\title{
Marketing promotion strategy of liquid foundation products based on university market -- Taking CHCEDO as an example
}

\author{
Qi Zhang ${ }^{1 *}$, Xinze $\mathrm{Li}^{2}$ \\ ${ }^{12}$ School of Economics, Wuhan University of Technology, Wuhan, China
}

\begin{abstract}
This article takes CHCEDO as an example to analyze the marketing promotion strategy of the foundation liquid products. From the two perspectives of industry background and market demand, we explore the marketing promotion strategy of the foundation liquid products in Colleges and universities. Finally, we summarize the academic marketing theory model of the liquid foundation products, and provide suggestions for developing the online and offline marketing of the powder liquid industry enterprises in Colleges and universities.
\end{abstract}

\section{Industry background and market demand analysis}

\subsection{Industry analysis}

With the upgrading and change of the public consumption concept and aesthetic concept, more and more consumers begin to pay attention to their external image. ${ }^{[1]}$ As the key of the whole makeup feeling, the bottom make-up has been highly concerned by the color makeup people for a long time. In 2019, the scale of China's color makeup market is about 55.2 billion yuan, and the scale of facial makeup market accounts for $48 \%$ of the color makeup market. Base makeup products are the largest market segment of facial makeup in China, accounting for about $90 \%$. In 2019, the market scale of China's bottom makeup products has reached 24.1 billion yuan.

Table 1: China primer products market size, 2015-2019 (\$billion) data source: prospective industry research institute

\section{China Primer Products Market Size, 2015-2019 (\$̦billion)}

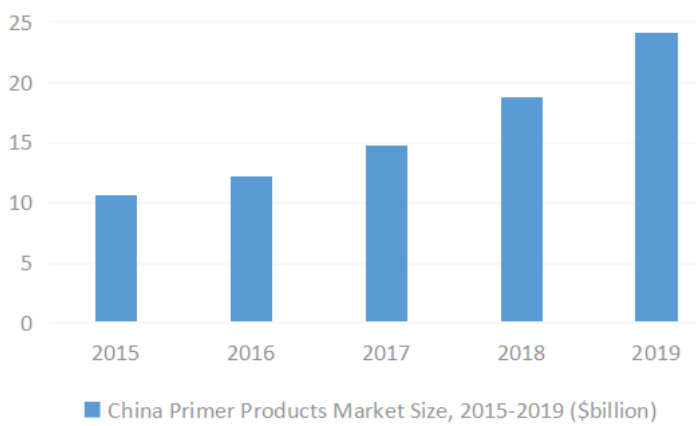

In terms of industry pain, the following two aspects are mainly manifested in the following aspects: first, the quality of service in the liquid foundation industry is difficult to control, resulting in frequent quality problems; second: the foundation industry is also fiercely competitive and homogenization is serious.

\subsection{Market demand}

\subsubsection{Foundation liquid industry}

Foundation liquid is an important subdivision product of the bottom makeup products. It has the function of concealing defects and improving skin color, and is very popular with consumers. With the rapid development of the bottom makeup market, the liquid foundation has become the largest market for the bottom makeup, with a market share of around $27 \%$ and a strong momentum of development. ${ }^{[2]}$

Table 2: the percentage of factors influencing consumers to buy foundations Data source: guanyantianxia

\section{The percentage of factors influencing consumers to buy foundations}

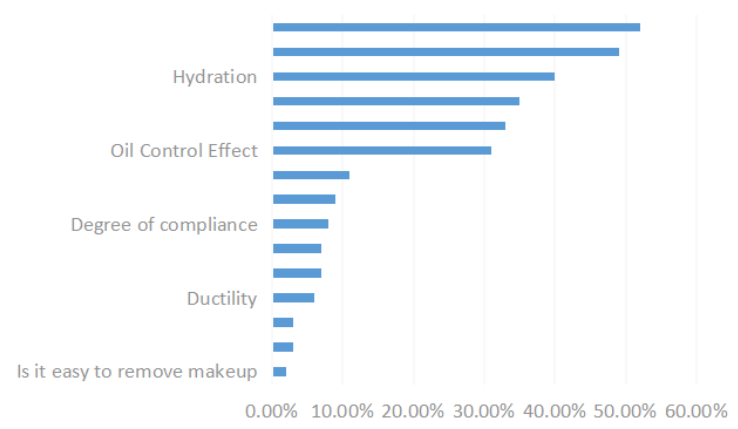

Focusing on the consumption market of colleges and universities, the post-95 college students have personalized and diversified consumption needs, and

\footnotetext{
* Corresponding author: 976490801@qq.com
} 
their acceptance of high-end beauty products is also increasing, showing a huge consumption potential.

The consumption characteristics of post-95 groups are mainly shown in the following aspects:

First, based on the interest of social networking, consumption circle trend is obvious; second, the pursuit of personality and emotional satisfaction; third, keen to pay for interests and idols.

\subsubsection{University market analysis}

China's economic development has entered a new normal, and consumption has become the core driving force of economic growth. ${ }^{[3]}$ College students grow up in the era of rapid development of the Internet, and their consumption and entertainment have obvious Internet marks. In 2018, the annual consumption scale of college students reached 380 billion yuan. At the same time, with the integration of the Internet into the daily life of college students, the Internet has also changed the way of College Students' social and entertainment. Contemporary college students grow up in a more mature Internet era and become the first generation of Internet aborigines. Among them, $66 \%$ of female college students consume daily chemical products, and only $1.1 \%$ of female college students in 2018 have never used cleansing and skin care products. In addition, the proportion of female students in cleaning, sunscreen, facial mask, face cream and lip balm was quite similar. Most girls began to use a full range of basic skin care products.

This shows that there is a great market space for beauty products in Colleges and universities, and the marketing of base makeup products is feasible.

\subsection{Brand and product analysis}

Nature hall, a product of Galan (Group) Co., Ltd., was founded in Shanghai in 2001. Based on the Chinese philosophy of the unity of man and nature, it advocates the concept of enjoying nature and beautiful life, develops and selects rare natural ingredients according to the characteristics of Chinese culture, diet and skin, integrates advanced technology, and is committed to providing better and more professional products and services for Chinese consumers.

Natural hall has a high brand awareness, but few consumers and loyal users have actually used its products, and the user viscosity is low, and consumers have certain prejudice against domestic products.

In terms of brand image, "domestic products", "old brands", "high performance price ratio" and the relevant spokesmen of nature hall are its prominent labels. In addition, the advanced technology of nature hall can make the makeup more lasting. It has added natural skin care ingredients, and the product types are rich, which can better meet the needs of users. The brand slogan "you are beautiful" has a high popularity, and the rise of domestic products makes consumption Their impression of domestic products has improved. However, the product homogeneity is serious, and the competition in the industry is fierce.

\section{Analysis of marketing promotion strategy}

\subsection{Core strategy analysis}

Based on the following pain points of users: 1. There is a certain prejudice against women's appearance in society; 2. The individual needs of "be yourself" are not satisfied; 3. Self is lost in all kinds of makeup gimmicks.

We can explore the two natural and psychological needs:

1. Users expect to achieve a harmonious state with their bodies;2. Users expect that a product can help their hearts stronger.

Therefore, we can put forward the following two solutions:

1. Put forward the product slogan "real yourself"; 2. Tell the product story, improve the style of the product, and help users tap their own characteristics and charm.

Therefore, we put forward the final positioning of the product: "powder yourself". Based on the previous interpretation, this slogan can be interpreted as three meanings: first, let users be their fans; second, users can tap their own characteristics and charm with this product; third, users become loyal users of the product.

The product is targeted at young women aged 18-23. Emotional positioning is to enable users to discover themselves, to be their fans, to show the beauty of nature, and to believe that they are born with unique charm. The function is positioned in Himalayan natural ingredients, creating 24-hour beauty.

\subsection{Product marketing activities}

\subsubsection{Outdoor advertising}

In recent years, the linkage of Chinese cultural landmarks, the depth of Chinese traditional culture to play the national trend of cross-border, through a wealth of interesting scene interaction to express, these practices not only bring consumers a refreshing sense of view, but also enhance the overall emotional added value and style of the product ${ }^{[4]}$ Because Wuhan is the city with the largest number of college students in China. Here we take Wuhan as an example.

The main form of outdoor advertising is various posters. The target locations are bus and subway stations. These places have a large flow of people. Outdoor presentation is conducive to creating an atmosphere and attracting people's attention. Liu Lingzi, the "super model girl group" and Huang cancan, the "Cherry Blossom goddess" and music teacher Hua Yuchen were invited to show their different images and attitudes from the impression, shoot theme posters, and more effectively convey to consumers the idea of "refusing to be defined" and resisting stereotypes. It helps to 
strengthen the output of brand awareness and value, and expand the promotion of ice cream foundation.

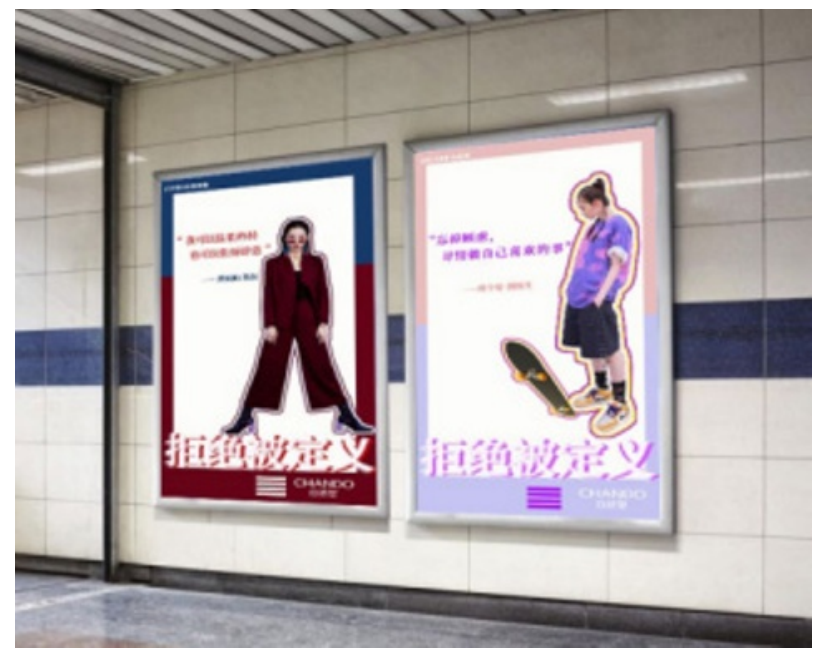

Fig. 1: billboard display

\subsubsection{Make up lesson 1}

Most of the female students who are about to enter the university are interested in make-up, but they may not be able to start because of many restrictions. Nature hall is willing to be a guide on the learning path of Xiaobai beauty. From the basic knowledge of beauty to the real experience of offline flash shops, they can easily realize the introduction of make-up, and at the same time, establish a good impression on the brand of nature hall. In addition, the setting of "special tea shop" will attract you through the setting of "young tea shop".

The course includes two aspects: interactive video and offline flash store.

In terms of interactive video, different from the high-level beauty technology video, the nature Hall official account launched the first lesson of powder making\# Topic: in the Bilibili series of interactive videos, the text of the embarrassment written by the newcomer's makeup is used as a video preview, and then the basic beauty problems such as the color number of the foundation solution and how to choose different skin are introduced. The first lesson is to share the embarrassment of your first makeup, that is, you have the chance to get the foundation liquid experience.

In terms of offline flash stores, we have released the activity preview in the campus we media in the early stage. After explaining the knowledge of base make-up purchase at the activity site, the make-up artist designed the make-up according to the personal appearance characteristics, and the photographer took photos on site after finishing the make-up. Participants take the topic \# beauty lesson 1 \# send photos and experience as the final assignment. Dignified and imposing on official account and administrative micro blog.

\subsubsection{Online activities}

First of all, it is the transformation of ordinary people. The official CHCEDO WeChat official account and official account number on campus are put online, and then the offline transformation is carried out. Starting from the daily life of consumers, the CHCEDO official WeChat official account has the advantage of large flow and high interaction. It promotes products through offline transformation activities, aiming to narrow the distance between young people and CHCEDO, and encourage users to independently spread and enhance brand sentiment.

On the implementation side, the official account is placed on the campus famous public number. The applicants need backstage messages to introduce their height and weight, hobbies, makeup requirements and knowledge of CHCEDO. The nature hall invites professional stylists to make up their hair to create a unique style for each girl. The photographer takes personal photos for the effect of makeup. Participants in the ordinary people's transformation activities were given the posters and exclusive official account tweets of "born confident".

In addition, there are also $\mathrm{H} 5$ games and other forms. Recently, idol manufacturing talent shows are particularly popular, with strong topic. Different from the entity of the draft star, pick's object is turned into a virtual self. Through a series of picking behaviors, girls can accept their imperfections in a vivid and interesting way. They also express their ideas through anti black links, and confront the body shaping from the outside to enhance their self-confidence. Taking advantage of the characteristics of wechat platform, such as large flow, strong interaction, simple operation and convenient sharing with $\mathrm{H} 5$, the girls will have a dream of virtual women's group.

\section{Marketing model summary}

\subsection{Online marketing model}

In the above-mentioned online marketing means, we can summarize the following models:

With product positioning as the core, relying on the layout of new media matrix, multi-party cooperation makes IP produce linkage effect, so as to improve the exposure and user attention.

Table 3: online marketing model

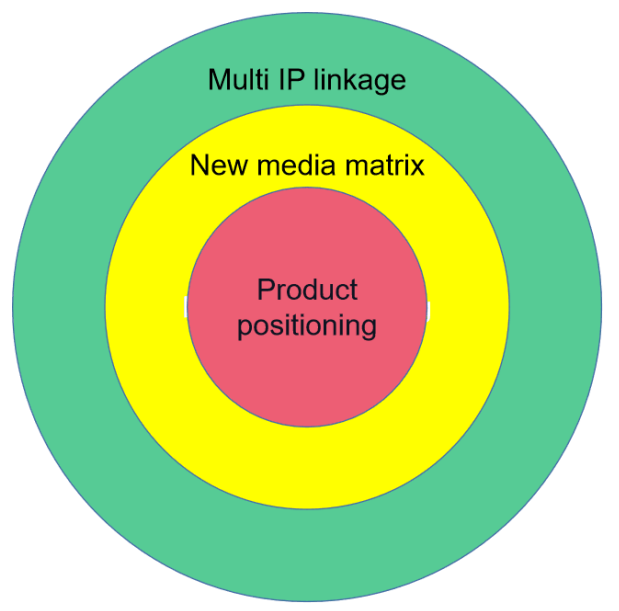




\subsection{Offline marketing model}

Offline scene marketing, to create a product scene immersion experience, to establish a more in-depth product impression of users, improve product purchase rate. Taking the product positioning as the core, based on the fashionable elements, it establishes a multi-dimensional marketing scene and carries out collision combination.

Table 4: offline marketing model

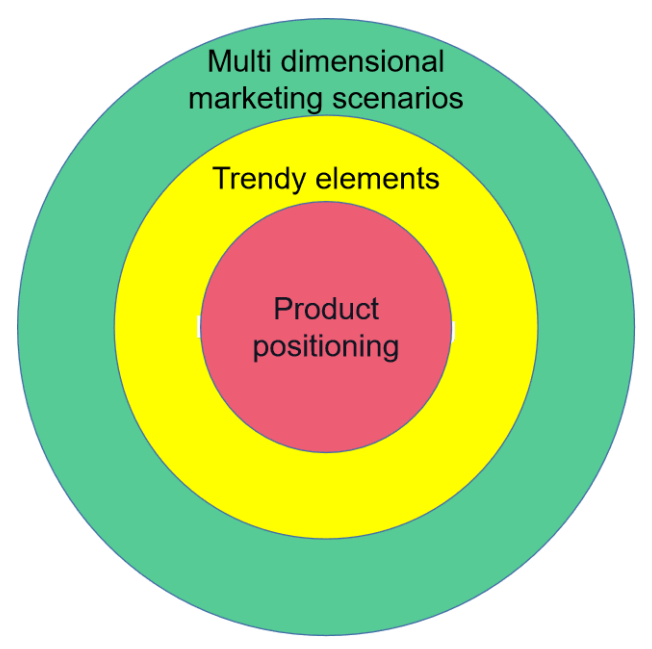

\subsection{Integrated marketing model}

It is not difficult to see that the establishment of cosmetics marketing model in university market is based on product positioning, and based on multi-party joint marketing method, it provides users with multi-dimensional immersive marketing experience.

Table 5: integrated marketing model

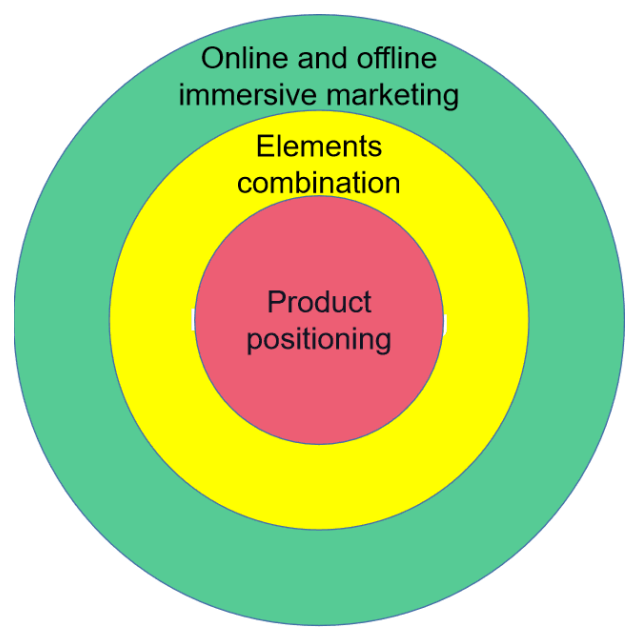

\section{Conclusion}

In an era where cosmetics are prevalent, cosmetics are facing huge challenges. ${ }^{[5]}$ The marketing of beauty products in Colleges and universities must take product positioning as the core, and need to improve the product style through the background story, and the products should be more universal and cost-effective. On this basis, the combination of multiple tidal current elements should be carried out flexibly, and the best way is to carry out cross-border integration. And use all possible forms to provide users with IP based immersive marketing experience. The university market has strong consumption ability, but the consumption choice is also very diverse. It is difficult for the new homogeneous products to become popular and grow rapidly. It is necessary to be able to move the positioning and price of consumers, as well as appropriate marketing.

\section{Reference:}

1. IResearch, super curriculum. Consumer insight report of college students in 2018 [R]. IResearch. 2018

2. "Hot words related to nature hall" [EB / OL] . https://www.wrd.cn/goSearch.shtml. 2020-12-21/2020-12-21

3. Qingyan. Foundation market report, [R].Qingyan. 2019

4. Natural Hall $\mathrm{x}$ Temple of Heaven: A National Tide Tribute, Full of Luck $[\mathrm{J}]$. Lin Ying. China Advertising. 2021(03)

5. Exploring the marketing environment and strategy of Naturally Hall cosmetics - taking Garan Naturally Hall cosmetics as an example [J]. Zhang Mingya. National business situation (theoretical research). 2013(08) 